\title{
亚麻响应盐、碱胁迫的生理特征
}

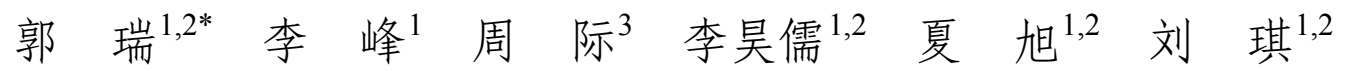

${ }^{1}$ 中国农业科学院农业环境与可持续发展研究所, 北京 $100081 ;{ }^{2}$ 农业部早作节水农业重点实验室, 北京 $100081 ;{ }^{3}$ 国土资源部土地整治中心, 北京 100034

摘 要 利用中性盐 $\mathrm{NaCl} 、 \mathrm{Na}_{2} \mathrm{SO}_{4}$ 和碱性盐 $\mathrm{NaHCO}_{3} 、 \mathrm{Na}_{2} \mathrm{CO}_{3}$ 混合模拟不同强度的盐、碱胁迫条件, 对亚麻(Linum usitatissimum)进行14天胁迫处理, 测定其地上部分和根生长速率、光合特征、离子平衡及有机渗透调节物质积累, 以探讨亚麻对 盐、碱两种胁迫的生理响应特点。研究表明: 亚麻生长对盐、碱胁迫的响应存在差异, 在相同盐浓度下, 碱胁迫对亚麻的伤 害大于盐胁迫。碱胁迫使地上部分中 $\mathrm{Na}^{+}$浓度急剧增高, 造成叶绿体破坏、光合色素含量下降, 光合能力及碳同化能力也急剧 下降。亚麻中 $\mathrm{Na}^{+}$含量随着胁迫强度的增加而升高, 而 $\mathrm{K}^{+}$含量呈下降趋势, 碱胁迫下的变化明显大于盐胁迫。因此, 碱胁迫导 致 $\mathrm{Na}^{+}$过度积累可能是碱胁迫对植物伤害大于盐胁迫的最主要原因。碱胁迫下 $\mathrm{Ca}^{2+}$ 和 $\mathrm{Mg}^{2+}$ 在根中下降明显, 可见高 $\mathrm{pH}$ 值阻碍 根对 $\mathrm{Ca}^{2+}$ 和 $\mathrm{Mg}^{2+}$ 的吸收。 $\mathrm{Fe}^{2+}$ 和 $\mathrm{Zn}^{2+}$ 对渗透调节的影响不大, 因为它们的离子含量较低。盐胁迫促进阴离子 $\left(\mathrm{Cl}^{-} 、 \mathrm{H}_{2} \mathrm{PO}_{4}{ }^{-}\right.$和 $\mathrm{SO}_{4}{ }^{2-}$ )的积累来平衡大量涌入的 $\mathrm{Na}^{+}$, 但是碱胁迫明显减少无机阴离子含量, 可能造成严重营养胁迫(如 $\mathrm{P}$ 和 $\mathrm{S}$ 不足)。亚麻在盐 胁迫下积累大量可溶性糖来平衡大量的 $\mathrm{Na}^{+}$, 但碱胁迫下积累大量有机酸来维持细胞内离子平衡和 $\mathrm{pH}$ 值稳定, 碱胁迫大量积 累的有机酸也可能被分泌到根外调节根外的 $\mathrm{pH}$ 值, 这说明亚麻对两种不同胁迫的响应方式不同。研究证明高 $\mathrm{pH}$ 值会直接影 响植物根系的生长发育, 影响植物矿质元素的吸收, 阻碍离子稳态重建, 有机酸代谢是亚麻碱胁迫下的关键适应机制。

关键词 亚麻; 盐胁迫; 碱胁迫; 生长特性; 光合特性; 离子平衡; 渗透调节

引用格式: 郭瑞, 李峰, 周际, 李吴儒, 夏旭, 刘琪 (2016). 亚麻响应盐、碱胁迫的生理特征. 植物生态学报, 40, 69-79. doi: 10.17521/cjpe.2015.0240

\section{Eco-physiological responses of linseed (Linum usitatissimum) to salt and alkali stresses} GUO Rui ${ }^{1,2^{*}}$, LI Feng ${ }^{1}$, ZHOU Ji $^{3}$, LI Hao-Ru ${ }^{1,2}$, XIA Xu ${ }^{1,2}$, and LIU Q ${ }^{1,2}$

${ }^{1}$ Institute of Environment and Sustainable Development in Agriculture, Chinese Academy of Agricultural Sciences, Beijing 100081, China; ${ }^{2}$ Key Laboratory of Dryland Agriculture, Ministry of Agriculture, Beijing 100081, China; and ${ }^{3}$ Land Consolidation and Rehabilitation Centre, Ministry of Land and Resources of the People's Republic of China, Beijing 100034, China

\section{Abstract}

Aims Effects of salt and alkali stresses $\left(\mathrm{NaCl}-\mathrm{Na}_{2} \mathrm{SO}_{4}\right.$ and $\left.\mathrm{NaHCO}-\mathrm{Na}_{2} \mathrm{CO}_{3}\right)$ were compared on growth, photosynthesis characters, ionic balance and osmotic adjustment of linseed (Linum usitatissimum), to elucidate the mechanisms of salt and alkali stress (high $\mathrm{pH}$ value) damage to plants, and their physiological adaptive mechanisms to the stresses.

Methods The experiment was carried out in an artificial greenhouse. Plants grew at approximately 700 $\mathrm{mmol} \cdot \mathrm{m}^{-2} \cdot \mathrm{s}^{-1}$ photosynthetic photon flux density $(P P F D)$ in greenhouse under photoperiod of $15 \mathrm{~h}$ in light and $9 \mathrm{~h}$ in dark. In each plastic pot (17 cm diameter) which contained $2.5 \mathrm{~kg}$ of washed sand, 20 linseed seeds were sown. The seedlings were exposed to stresses lasting 14 days after 2 months.

Important findings The inhibitory effects of alkali stress on linseed growth were more remarkable than those of salt stress, indicating that alkali and salt represent two distinct forms of stress. The alkali stress increased the $\mathrm{Na}^{+}$ content in shoots, damaged the photosynthetic system, and highly reduced the net photosynthetic rate and $\mathrm{C}$ assimilation capacity. Under salinity stress, the $\mathrm{Na}^{+}$content increased, the $\mathrm{K}^{+}$content decreased with increasing stress. Greater changes were observed under alkali than under salt stress. Alkali stress caused the massive influx of $\mathrm{Na}^{+}$, which probably explained that the harmful of alkali stress on plants was stronger than that of salt stress. Under alkali stress, $\mathrm{Ca}^{2+}$ and $\mathrm{Mg}^{2+}$ decreased in roots, showing that high $\mathrm{pH}$ value around roots hindered the absorption of them. $\mathrm{Fe}^{2+}$ and $\mathrm{Zn}^{2+}$ had little effects on the osmotic adjustment, mainly because of they had a low ion content. Under salt stress, anion increased in order to balance the sharp increase of $\mathrm{Na}^{+}$. However, alkali stress made severe deficit of negative charge, broke the intracellular ionic balance and $\mathrm{pH}$ homeostasis, and caused a

收稿日期Received: 2015-06-24 接受日期Accepted: 2015-12-27

*E-mail: guorui01@caas.cn 
series of strain response. Our results showed that linseed enhanced the synthesis of soluble sugars to balance massive influx of $\mathrm{Na}^{+}$under salt stress, but linseed enhanced the synthesis of organic acids to compensate for the shortage of inorganic anions, which might be a key pathway for the $\mathrm{pH}$ adjustment. In conclusion, the alkali stress (high $\mathrm{pH}$ value) clearly inhibited the growth, element absorption, ion homeostasis reconstruction of plants. Organic acid concentration is possibly a key adaptive factor for linseed to maintain intracellular ion balance and regulate high $\mathrm{pH}$ value under alkali stress.

Key words Linum usitatissimum; salt stress; alkali stress; growth characters; photosynthesis characters; ionic balance; osmotic adjustment

Citation: Guo R, Li F, Zhou J, Li HR, Xia X, Liu Q (2016). Eco-physiological responses of linseed (Linum usitatissimum) to salt and alkali stresses. Chinese Journal of Plant Ecology, 40, 69-79. doi: 10.17521/cjpe.2015.0240

全世界盐碱地面积已经超过总陆地面积的 $6 \%$ 和农业灌溉面积的 $20 \%$, 预计到2050年, 将会有超 过 50\%的耕地盐渍化(张建锋等, 2005)。我国耕地中 盐碱地约占 $15 \%$, 另外还有 2000 多万 $\mathrm{hm}^{2}$ 的盐荒地 (李涁等, 2005)。根据我国土壤特性和所含盐分特点, 将其分为盐土和碱土两大类, 其中最主要的致害离 子为 $\mathrm{Na}^{+} 、 \mathrm{Cl}^{-} 、 \mathrm{HCO}_{3}{ }^{-}$和 $\mathrm{CO}_{3}{ }^{2-}$, 这些离子对植物的 胁迫作用, 除了包括直接的胁迫效应外, 还包括离 子间复杂的相互作用(石德成等, 2002)。

盐胁迫主要是渗透胁迫和离子毒害, 它影响各 种离子在植物细胞内的分布, 破坏胞内离子平衡, 植物必须进行渗透调节并在细胞内重建离子稳态 (Zhang et al., 2010; Zhang \& Shi, 2013)。碱胁迫与盐 胁迫相比, 除了渗透胁迫和离子毒害外, 还涉及高 pH值伤害(Yang et al., 2008)。植物应对碱胁迫, 不 仅要进行细胞内 $\mathrm{pH}$ 值调节以保持离子平衡, 同时 还必须付出大量的物质和能量来调节根际微环境 的pH值(Shi \& Wang, 2005)。所以渗透调节、细胞 内离子平衡、根外 $\mathrm{pH}$ 值调节是植物抗碱胁迫的关 键所在。

一直以来, 国内外抗盐方面的研究较多考虑单 一盐 $(\mathrm{NaCl})$ 对植物的影响, 主要涉及胁迫生理、表 观遗传、离子转运、激素调节、基因表达等方面(孔 令安等, 2000; Lu et al., 2013; Wu et al., 2013)。而土 壤碱胁迫这一严重的环境问题仍未得到研究者的充 分重视, 仅有少量描述性报道涉及植物抗碱生理及 分子机制(萨如拉等, 2014; 张一弓等, 2014)。只有区 别研究盐胁迫和碱胁迫, 才能真正揭示植物抗盐碱 机制, 为最终培育出新的抗盐碱植物品种打下基础, 为盐碱化地的恢复和治理提供理论支持。

亚麻(Linum usitatissimum)不仅是重要的油料作 物, 还是一种工业原料。亚麻油富含不饱和脂肪酸
和对人体有益的多种营养物质, 另外, 亚麻的天然 纤维具有吸汗、透气性良好和对人体无害等特点 (McKenzie \& Deyholos, 2011)。目前, 有关亚麻盐胁 迫下的生理响应研究不多, 且多集中在单一盐 $(\mathrm{NaCl})$ 方面, 其对碱胁迫的响应及适应机制国内外 未见相关报道(Ashraf \& Fatima, 2004; Chemikosova et al., 2006)。

考虑到大多数内陆盐碱地的盐分组成特点, 本 研究将 2 种中性盐 $\left(\mathrm{NaCl}\right.$ 和 $\left.\mathrm{Na}_{2} \mathrm{SO}_{4}\right)$ 和 2 种碱性盐 $\left(\mathrm{NaHCO}_{3}\right.$ 和 $\left.\mathrm{Na}_{2} \mathrm{CO}_{3}\right)$ 分别按 $1: 1$ 摩尔比混合, 分别模 拟不同强度的盐和碱胁迫条件处理亚麻, 研究亚麻 在盐、碱胁迫下的生理生态响应机制, 揭示盐胁迫 和碱胁迫的异同点, 同时为亚麻抗盐碱品种的篎选 和鉴别提供一定的理论依据。

\section{1 材料和方法}

\section{1 实验材料}

亚麻品种‘宁夏-15’。

\section{2 模拟胁迫}

根据我国内陆盐碱化土壤主要盐分组成以及盐 度、碱度复杂多变的特点。选取 $\mathrm{NaCl} 、 \mathrm{Na}_{2} \mathrm{SO}_{4}$ 、 $\mathrm{NaHCO}_{3}$ 和 $\mathrm{Na}_{2} \mathrm{CO}_{3}$, 模拟土壤盐碱胁迫环境, 其中 $\mathrm{NaCl} 、 \mathrm{Na}_{2} \mathrm{SO}_{4}$ 为中性盐, $\mathrm{NaHCO}_{3} 、 \mathrm{Na}_{2} \mathrm{CO}_{3}$ 为碱性 盐, 均按摩尔比 $1: 1$ 混合。利用便携式电导仪(DDG2080-S, 博取公司, 上海)、露点水势仪(Wescor Psypro, Wescor, Utah, USA)和数字 $\mathrm{pH}$ 计 (PHS-3C, 虹益公司, 上海) 分别测定各种浓度处理液的电导 率、渗透势和 $\mathrm{pH}$ 值。

\section{3 材料培养}

挑选整齐一致的亚麻种子播种于直径 $17 \mathrm{~cm}$ 的 塑料花盆内, 每盆装 $2.5 \mathrm{~kg}$ 洗净的细砂。共种植 40 盆, 每盆定苗5株, 出苗后每天用 0.5 倍Hoagland营养液 
进行浇灌, 培养 2 个月。整个实验在室外进行, 并进 行人工遮雨, 白天温度为 $27.0-21.5{ }^{\circ} \mathrm{C}$, 夜晚为 $21.5-$ $18.5{ }^{\circ} \mathrm{C}$ 。 4 周后, 将 40 盆分为 8 组, 每组为 5 盆, 一盆 为一个重复。有 1 组作为胁迫处理前基础生物量指标 的测试样品。剩下的 7 组, 1 组为对照组, 每天仍浇 0.5 倍Hoagland营养液; 其他6组按照设计的不同浓度 对亚麻幼苗进行连续14天的胁迫处理。第 15 天对实 验样品进行取样, 将根和地上部分从子叶痕处分离 开来。称鲜质量后, 将植株置于 $80{ }^{\circ} \mathrm{C}$ 烘箱内杀青 15 $\min$, 杀青后将植株转置 $40{ }^{\circ} \mathrm{C}$ 的真空干燥器内烘干 至恒质量, 称取干质量并记录。将样品磨成粉末, 用 于测定各种溶质含量。

\section{4 生理指标测定}

\subsection{1 生长指标的测定}

相对生长率 $(\mathrm{RGR})=(\ln$ 处理后干质量 $-\ln$ 处理 前干质量)/处理天数(Kingsbury et al., 1984)

绝对含水量 $(\mathrm{AWC})=($ 鲜质量 $-干$ 质量 $)$ / 鲜质量, 单位为 $\mathrm{mg} \cdot \mathrm{g}^{-1}$ 。

\subsection{2 光合特性指标的测定}

在取样前利用苂光仪(IMAGING-PAM，WALZ， Effeltrich, Germany)测定叶绿素苂光参数: 光系统II (PSII) 原初光能转换效率 $\left(\Phi_{\mathrm{PSII}}\right)$ 、非光化学淬灭 $(N P Q)$ 、光化学淬灭系数 $\left(q_{\mathrm{P}}\right)$, 各参数数值均是在选 定模式下系统自动计算生成。测定前, 叶片暗适应 $20 \mathrm{~min}$, 每个处理测定 5 个叶片, 所有操作均在背景 光强低于 $1 \mu \mathrm{mol} \cdot \mathrm{m}^{-2} \cdot \mathrm{s}^{-1}$ 的环境中进行。仪器的饱和 脉冲光的强度为 $2400 \mu \mathrm{mol} \cdot \mathrm{m}^{-2} \cdot \mathrm{s}^{-1}$, 光化光强设为 默认值 $185 \mu \mathrm{mol} \cdot \mathrm{m}^{-2} \cdot \mathrm{s}^{-1}$, 测量光低于 $0.5 \mu \mathrm{mol} \cdot \mathrm{m}^{-2}$. $\mathrm{s}^{-1}$ (Genty et al., 1989)。

待苂光测量完毕后, 将叶片采下, 洗净磨碎, 称取样品 $0.1 \mathrm{mg}$, 用体积比 $1: 1$ 的丙酮和无水乙醇作 为萃取液进行萃取。在波长440、645和663 nm下测 定吸光度, 每一个萃取实验要进行 5 次重复。应用 Arnon (1949)方程计算叶绿素含量。

\subsection{3 无机溶质指标的测定}

使用离子色谱系统(DX-300, Thermo Fisher Scientific, Massachusetts, USA)、色谱柱 (AS4A-SC, Dionex, Sunnyvale, USA)和电导检测器 (CDM-II, Dionex, Sunnyvale, USA) 测定。使用 $\mathrm{Na}_{2} \mathrm{CO}_{3}$ : $\mathrm{NaHCO}_{3}=1.7: 1.8\left(\mathrm{mmol} \cdot \mathrm{L}^{-1}\right)$ 的混合溶液作为流动 相。测量阳离子 $\left(\mathrm{K}^{+} 、 \mathrm{Na}^{+} 、 \mathrm{Ca}^{2+}\right.$ 和 $\left.\mathrm{Mg}^{2+}\right)$ 和阴离子 $\left(\mathrm{NO}_{3}{ }^{-} 、 \mathrm{Cl}^{-} 、 \mathrm{SO}_{4}{ }^{2-}\right.$ 和 $\left.\mathrm{H}_{2} \mathrm{PO}_{4}^{-}\right)$含量。

\subsection{4 有机溶质指标的测定}

在 $70{ }^{\circ} \mathrm{C}$ 下利用 $70 \%$ 酒精对样品进行萃取, 并 参照鲍士旦(1981)使用葱酮方法测定可溶性糖含 量。利用 $\mathrm{HCl}\left(0.05 \mathrm{~mol} \cdot \mathrm{L}^{-1}\right)$ 在 $100{ }^{\circ} \mathrm{C}$ 下提取 $2 \mathrm{~h}$, 用高 压液相色谱法分析测定有机酸组分及含量, 方法参 照荆家海和丁钟荣(1981)。脯氨酸和甜菜碱的提取 和测定分别参考朱广廉等(1983)和Gorham等(1982) 的方法。

\section{5 数据分析}

利用SPSS 17.0和Excel对数据进行统计、分析及 绘图, 试验数据以平均值土标准误差表示, 每个数 据均是 5 次重复的平均值, 采用Duncan检测植物各 项生理参数的差异性, $p<0.05$ 被视为存在显著性 差异。

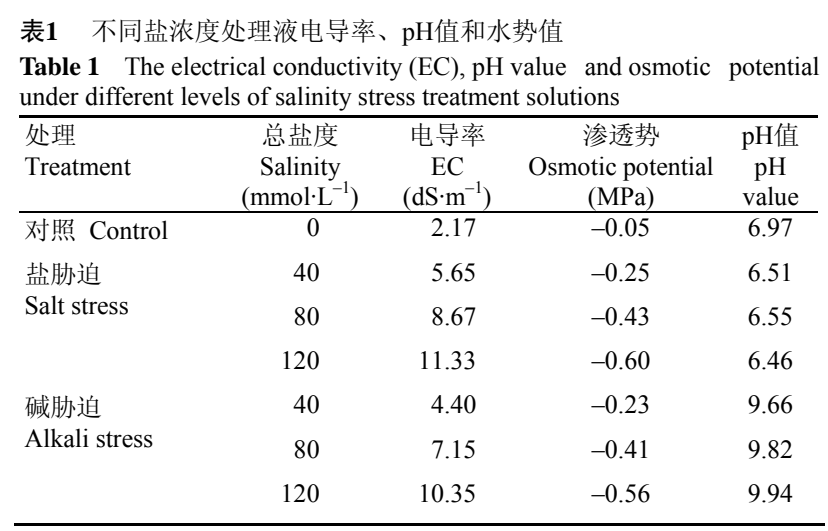

\section{2 结果}

\section{1 盐、碱胁迫对亚麻生长特性的影响}

当盐浓度大于 $40 \mathrm{mmol} \cdot \mathrm{L}^{-1}$ 时, 亚麻地上部分和 根RGR开始下降, 并随盐浓度增加而加速下降, 碱 胁迫的下降幅度均大于相同浓度的盐胁迫; 高碱胁 迫(120 mmol $\left.\cdot \mathrm{L}^{-1}\right)$ 下亚麻根RGR降到很低的水平(图 $1 \mathrm{~A} 、 1 \mathrm{~B}, p<0.05)$ 。盐、碱胁迫下亚麻地上部分、 根含水量均随胁迫强度增加而下降, 而碱胁迫下含 水量下降幅度明显大于盐胁迫下(图1C、1D, $p<$ $0.05)$ 。

\section{2 盐、碱胁迫对亚麻光合特性的影响}

与对照相比, 盐胁迫对亚麻叶片各项苂光参数 影响较小, 且差异不显著(图 $2 \mathrm{~A}-2 \mathrm{C}, p<0.05)$ 。但是 碱胁迫浓度大于 $80 \mathrm{mmol} \cdot \mathrm{L}^{-1}$ 时, 各项苂光参数变化 明显, $\Phi_{\mathrm{PSII}}$ 和 $q_{\mathrm{P}}$ 随胁迫强度增加而下降, 与其相对的 是 $N P Q$ 随胁迫强度增加而上升(图 $2 \mathrm{~A}-2 \mathrm{C}, p<0.05$ )。 

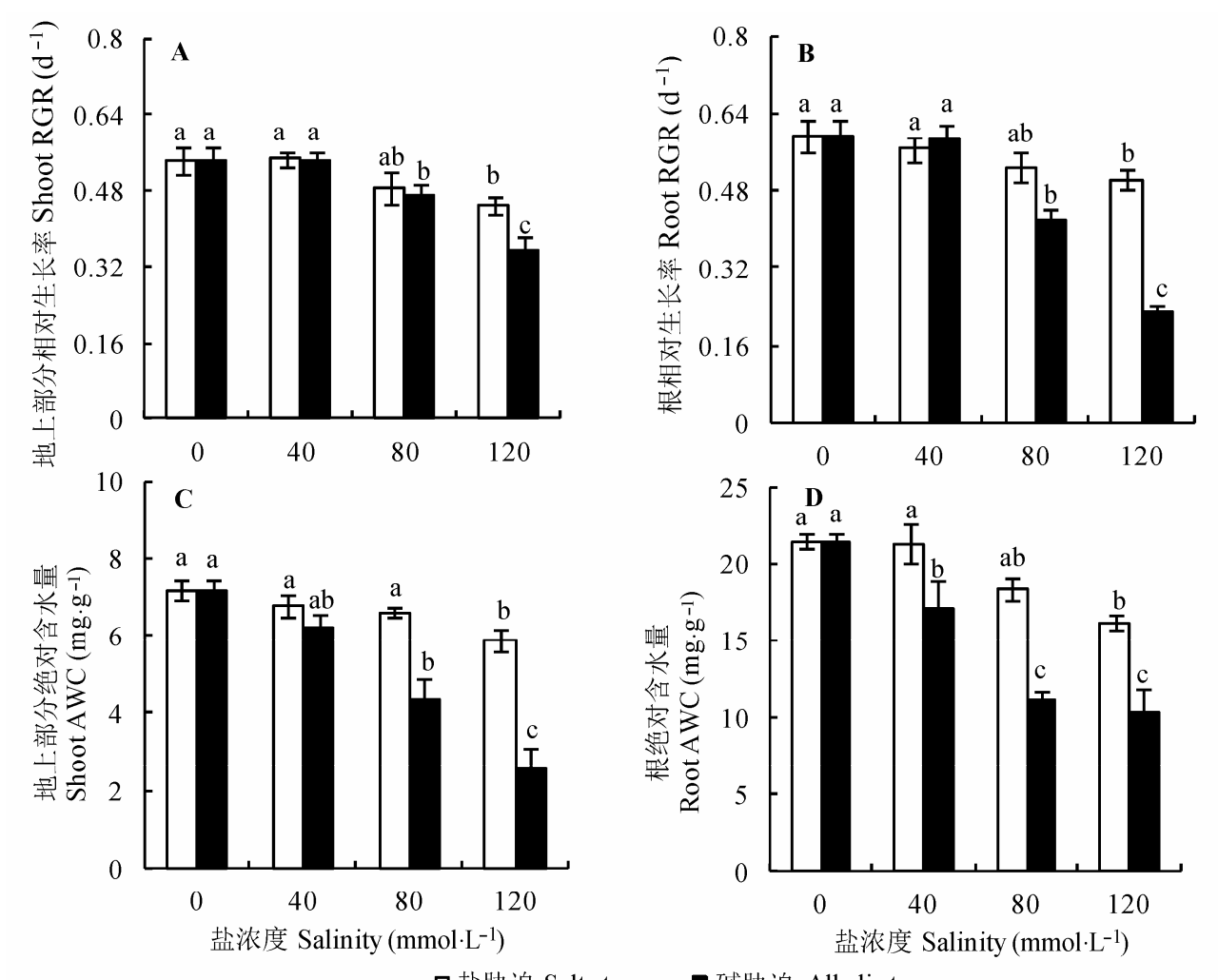

口盐胁迫 Salt stress

口碱胁迫 Alkali stress

图1 盐、碱胁迫对亚麻地上部分和根相对生长率(RGR)和绝对含水量(WC)的影响(平均值土标准误差, $n=5$ )。不同小写字母 表示不同处理间差异显著 $(p<0.05)$ 。

Fig. 1 Effects of salt and alkali stresses on shoot and root ground relative growth rate (RGR) and on absolute water content (AWC) (mean $\pm S E, n=5$ ). Different small letters indicate significant differences between different treatments $(p<0.05)$.

盐胁迫和中低度碱胁迫均没有影响亚麻叶绿素含量 的变化, 但高强度碱胁迫 $\left(120 \mathrm{mmol} \cdot \mathrm{L}^{-1}\right)$ 导致 3 种叶 绿素均急剧下降(图2D-2F, $p<0.05$ )。

\section{3 盐、碱胁迫对亚麻离子平衡的影响}

与对照相比，盐、碱胁迫对地上部分中 $\mathrm{K}^{+}$影响 较小, 只有在盐浓度大于 $80 \mathrm{mmol} \cdot \mathrm{L}^{-1}$ 后 $\mathrm{K}^{+}$含量才开 始下降。但是根中 $\mathrm{K}^{+}$含量下降明显, 且碱胁迫处理 下降程度明显大于盐胁迫(图 $3 \mathrm{~A}_{1} 、 3 \mathrm{~A}_{2}, p<0.05$ )。 地上部分和根中 $\mathrm{Na}^{+}$含量均随盐度增加呈增加趋势, 且碱胁迫下增加幅度明显高于盐胁迫(图 $3 \mathrm{~B}_{1} 、 3 \mathrm{~B}_{2}, p$ $<0.05)$ 。如图3所示, $\mathrm{Ca}^{2+}$ 和 $\mathrm{Mg}^{2+}$ 变化趋势基本一致。 地上部分中 $\mathrm{Ca}^{2+}$ 和 $\mathrm{Mg}^{2+}$ 含量在盐、碱胁迫下无显著 变化, 在根中 $\mathrm{Ca}^{2+}$ 和 $\mathrm{Mg}^{2+}$ 含量则随胁迫强度增加而 呈现下降趋势, 碱胁迫下降幅度明显大于盐胁迫 (图3 $\mathrm{C}_{1} 、 3 \mathrm{C}_{2}, 3 \mathrm{D}_{1} 、 3 \mathrm{D}_{2}, p<0.05$ )。与对照相比, 盐、 碱胁迫使地上部分、根 $\mathrm{K}^{+} / \mathrm{Na}^{+}, \mathrm{Ca}^{2+} / \mathrm{Na}^{+}$和 $\mathrm{Mg}^{2+} / \mathrm{Na}^{+}$ 大幅度下降, 且随盐度增加缓慢降低, 碱胁迫下的 下降幅度均大于盐胁迫(图 $3 \mathrm{E}_{1}-3 \mathrm{G}_{2}, p<0.05$ )。随着
盐度增加, 盐胁迫下亚麻地上部分和根中的 $\mathrm{Cl}^{-}$含 量均增加, 碱胁迫下 $\mathrm{Cl}^{-}$含量均下降, 根中的下降幅 度明显大于地上部分(图 $4 \mathrm{~A}_{1}$ 和 $\mathrm{A}_{2}, p<0.05$ )。盐、碱 胁迫下地上部分和根中 $\mathrm{NO}_{3}{ }^{-}$含量均低于对照, 根中 $\mathrm{NO}_{3}{ }^{-}$含量在碱胁迫下明显低于盐胁迫(图 $4 \mathrm{~B}_{1}$ 和 $\mathrm{B}_{2}, p$ $<0.05)$ 。随着胁迫强度增加, 盐胁迫均使地上部分 和根中 $\mathrm{H}_{2} \mathrm{PO}_{4}{ }^{-}$和 $\mathrm{SO}_{4}{ }^{2-}$ 含量增加, 但碱胁迫均导致 其含量降低, 尤其在根中(图 $4 \mathrm{C}_{1}-4 \mathrm{D}_{2}, p<0.05$ )。

\section{4 盐、碱胁迫对亚麻有机溶质的影响}

如图 $5 \mathrm{~A}_{1} 、 5 \mathrm{~A}_{2}$ 所示, 随着胁迫强度的增加, 亚 麻地上部分和根中可溶性糖含量在盐胁迫下呈现上 升趋势, 而碱胁迫下呈现下降趋势 $(p<0.05)$ 。地上 部分和根中有机酸在盐胁迫下或稍有减少或无明显 变化, 但碱胁迫下有机酸含量不仅明显高于相同强 度盐胁迫下, 而且均随盐度增加而明显上升(图5 $\mathrm{B}_{1}$, $\left.5 \mathrm{~B}_{2}, p<0.05\right)$ 。另外, 盐、碱胁迫均使脯氨酸含量增 加, 碱胁迫下增加幅度大于盐胁迫 $\left(5 \mathrm{C}_{1}, 5 \mathrm{C}_{2}, p<\right.$ $0.05)$ 。盐、碱胁迫下亚麻不但不积累甜菜碱, 反而 

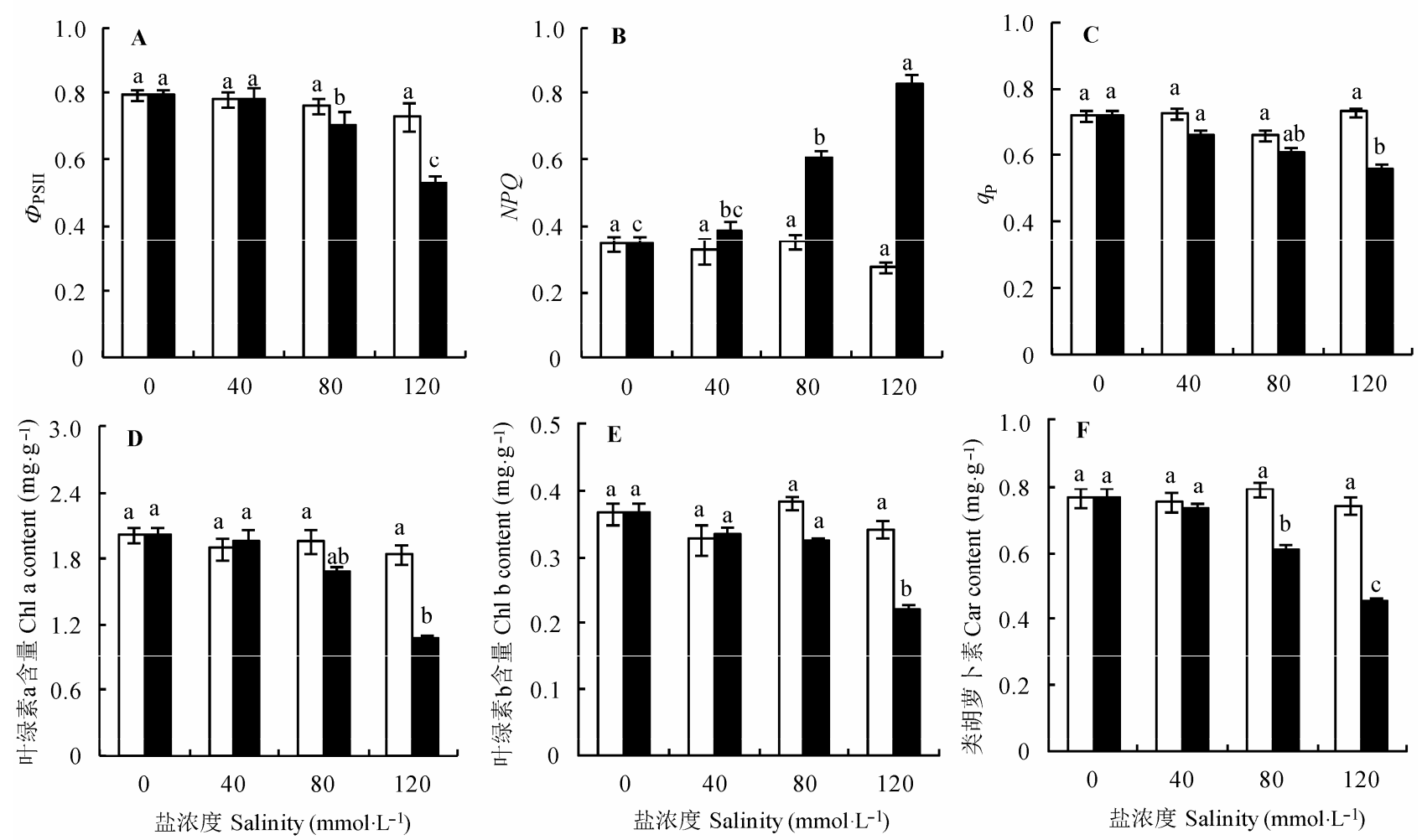

口盐胁迫 Salt stress

口碱胁迫 Alkali stress

图2 盐、碱胁迫对亚麻叶片光系统II 原初光能转换效率 $\left(\Phi_{\mathrm{PSII}}\right)$ 、非光化学淬灭 $(N P Q)$ 、光化学淬灭系数 $\left(q_{\mathrm{P}}\right)$ 、叶绿素a $(\mathrm{Chl} \mathrm{a})$ 、 叶绿素b $(\mathrm{Chl} b)$ 和类胡萝卜素 $(\mathrm{Car})$ 含量的影响(平均值标准误差, $n=5)$ 。不同小写字母表示不同处理间差异显著 $(p<0.05)$ 。

Fig. 2 Effects of salt and alkali stresses on the maximum PSII quantum yield $\left(\Phi_{\mathrm{PSII}}\right)$, non-photochemical quenching $(N P Q)$ and photochemical quenching $\left(q_{\mathrm{P}}\right)$ of chlorophyll a fluorescence, and chlorophyll a (Chl a), chlorophyll b (Chl b), carotenoids (Car) parameters in leaves of linseed seedlings (mean $\pm S E, n=5$ ). Different small letters indicate significant differences between different treatments $(p<0.05)$.

抑制其合成, 导致含量降低, 根中含量降低幅度大 于地上部分中 $\left(5 \mathrm{D}_{1}, 5 \mathrm{D}_{2}, p<0.05\right)$ 。

\section{3 讨论}

\section{1 盐、碱胁迫对亚麻生长特性的影响}

RGR是生命活动的最终体现, 可以作为植物对 外界环境胁迫的响应指标(Yang et al., 2008)。本实验 结果表明在相同盐浓度下, 碱胁迫RGR降幅大于盐 胁迫。碱胁迫对植物的伤害甚于盐胁迫的原因可能 与两种胁迫的作用机制有关, 盐胁迫主要是渗透胁 迫及离子毒害, 而碱胁迫除包括这两种胁迫作用外, 还包括高pH胁迫(石德成等, 2002)。当胁迫强度大于 $40 \mathrm{mmol} \cdot \mathrm{L}^{-1}$ 时, 碱胁迫(高 $\mathrm{pH}$ 值) 明显地抑制亚麻 RGR, 尤其是根系生长(图1)。该结果与抗碱植物明 显不同, 例如地肤(Kochia scoparia)和虎尾草(Chloris virgata) (Yang et al., 2008)。抗碱植物在高强度
碱胁迫下仍然可以保持较强的根RGR, 这表明植物 的根系对 $\mathrm{pH}$ 值的调节和适应能力, 可能是决定植物 体自身抗碱性的关键生理特性。AWC的变化可以反 映植物体受到外界环境胁迫时的基本生理反应。盐、 碱胁迫下亚麻AWC的变化趋势和RGR相似。结果充 分证明盐、碱胁迫属于不同胁迫类型, 碱胁迫对植 物构成的危害明显大于盐胁迫。得到的结论与以往 的一些报道相一致(颜宏等, 2000; 石德成等, 2002)。 高pH值会引起根系周围某些离子发生沉淀, 导致根 系附近离子失衡, 破坏根系对营养元素的吸收; 另 外, 高 $\mathrm{pH}$ 值可能会导致泡内质子减少, 进而破坏或 抑制根细胞跨膜电化学势梯度, 最终影响根系的正 常生理功能。

\section{2 盐、碱胁迫对亚麻光合特性的影响}

叶绿素荧光特性可以反映出外界胁迫环境对植 物光合器官的伤害程度, 其中PSII变化可以清楚显 

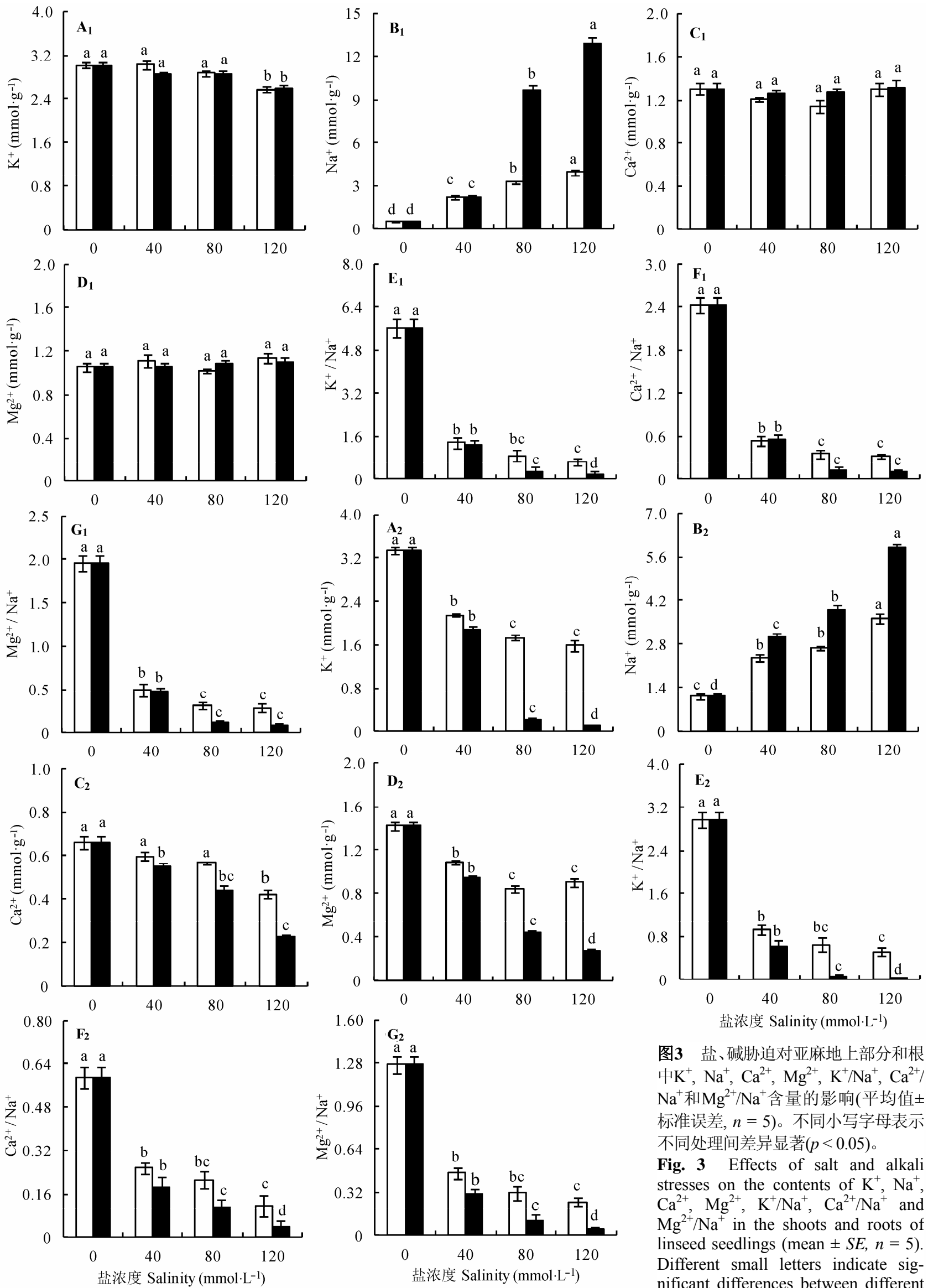

口盐胁迫 Salt stress

碱胁迫 Alkali stress

图3 盐、碱胁迫对亚麻地上部分和根 中 $\mathrm{K}^{+}, \mathrm{Na}^{+}, \mathrm{Ca}^{2+}, \mathrm{Mg}^{2+}, \mathrm{K}^{+} \mathrm{Na}^{+}, \mathrm{Ca}^{2+}$ $\mathrm{Na}^{+}$和 $\mathrm{Mg}^{2+} / \mathrm{Na}^{+}$含量的影响(平均值 \pm 标准误差, $n=5$ )。不同小写字母表示 不同处理间差异显著 $(p<0.05)$ 。

Fig. 3 Effects of salt and alkali stresses on the contents of $\mathrm{K}^{+}, \mathrm{Na}^{+}$, $\mathrm{Ca}^{2+}, \mathrm{Mg}^{2+}, \mathrm{K}^{+} / \mathrm{Na}^{+}, \mathrm{Ca}^{2+} / \mathrm{Na}^{+}$and $\mathrm{Mg}^{2+} / \mathrm{Na}^{+}$in the shoots and roots of linseed seedlings (mean $\pm S E, n=5$ ). Different small letters indicate significant differences between different treatments $(p<0.05)$. 

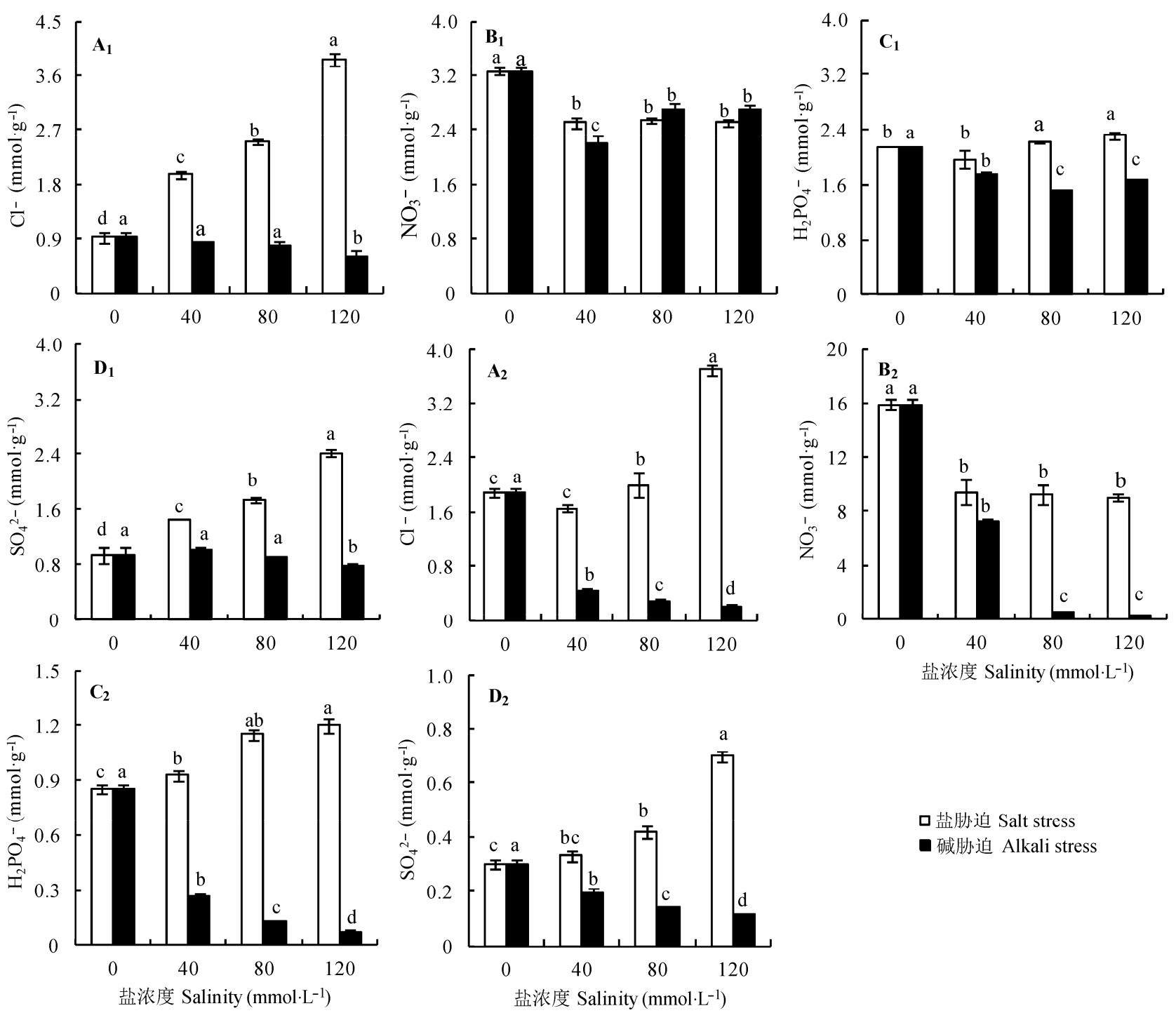

口盐胁迫 Salt stress

口碱胁迫 Alkali stress

图4 盐、碱胁迫对亚麻地上部分和根干物质中 $\mathrm{Cl}^{-} 、 \mathrm{NO}_{3}^{-} 、 \mathrm{H}_{2} \mathrm{PO}_{4}{ }^{-}$和 $\mathrm{SO}_{4}{ }^{2-}$ 含量的影响(平均值沶准误差, $n=5$ )。不同小写字 母表示不同处理间差异显著 $(p<0.05)$ 。

Fig. 4 Effects of salt and alkali stresses on the contents of $\mathrm{Cl}^{-}, \mathrm{NO}_{3}{ }^{-}, \mathrm{H}_{2} \mathrm{PO}_{4}{ }^{-}$and $\mathrm{SO}_{4}{ }^{2-}$ in the shoots and roots of linseed seedlings (mean $\pm S E, n=5$ ). Different small letters indicate significant differences between different treatments $(p<0.05)$.

示出外界环境对植物光合能力的影响程度(冯玉龙 等, 2001; 梁红柱等, 2004)。本研究发现, 盐胁迫下 亚麻叶片各项苂光参数并没有明显的变化, 这表明 盐胁迫对植物体自身的光合能力影响并不明显, 之 前的一些报道(Everard et al., 1994; Lu et al., 2002) 也证明了这一点。但是, 碱胁迫下PSII活性下降明 显, 表明碱胁迫引起了光抑制, 导致亚麻叶片的光 合能力降低, 主要原因可能是大量 $\mathrm{Na}^{+}$和高 $\mathrm{pH}$ 值共 同作用的结果。 $N Q P$ 属于光合系统的自我保护机制, 在碱胁迫下 $N Q P$ 的增加有利于提高光能利用效率, 增强光电子传递速率。 $q_{\mathrm{P}}$ 反映了光化学能量转换的
能力, 是反映光合速率高低的有效指标, 本实验结 果表明碱胁迫严重抑制了亚麻的光合效率(BolhàrNordenkampf et al., 1989)。叶绿素和类胡萝卜素是 高等植物的主要光合色素。叶绿素和类胡萝卜素的 积累在盐胁迫下变化不明显, 但是在碱胁迫下下降 明显。这可能是碱胁迫引起Mg沉淀从而抑制了叶绿 素和类胡萝卜素的合成(Reddy \& Vora, 1986; Shi \& Zhao, 1997)。另外, 也有可能是高pH值促进了叶绿 素分解酶的活性。

\section{3 盐、碱胁迫对亚麻离子平衡的影响}

$\mathrm{Na}^{+}$和 $\mathrm{K}^{+}$代谢是植物适应盐渍环境最为重要的 

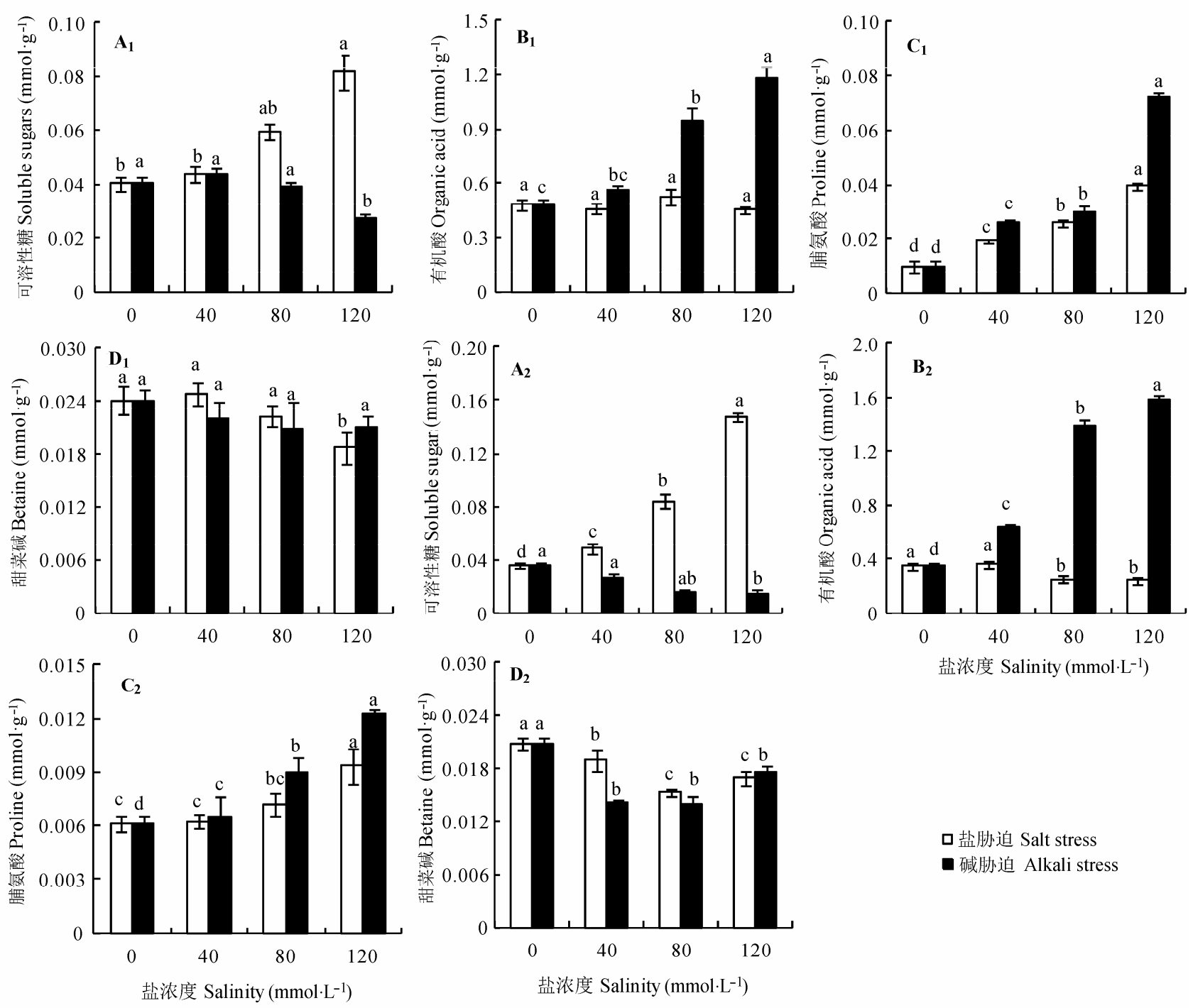

盐浓度 Salinity $\left(\mathrm{mmol} \cdot \mathrm{L}^{-1}\right)$

口盐胁迫 Salt stress

口碱胁迫 Alkali stress

图5 盐、碱胁迫对亚麻地上部分和根干物质中可溶性糖、有机酸、脯氨酸和甜菜碱含量的影响(平均值土标准误差, $n=5$ )。 不同小写字母表示不同处理间差异显著 $(p<0.05)$ 。

Fig. 5 Effects of salt and alkali stresses on the contents of soluble sugars, organic acid, proline and betaine in the shoots and roots of linseed seedlings (mean $\pm S E, n=5$ ). Different small letters indicate significant differences between different treatments $(p<0.05$ ).

生理代谢活动, $\mathrm{Na}^{+}$对于大多数植物而言是有害的, 当环境中 $\mathrm{Na}^{+}$含量增高时, 会拮抗植物对 $\mathrm{K}^{+}$的吸收, 从而限制植物生长(Brugnoli \& Lauteri, 1991; 王宁 等, 2015)。本研究发现, 盐、碱胁迫均造成亚麻地 上部分和根中 $\mathrm{Na}^{+}$含量升高, $\mathrm{K}^{+}$含量降低, 碱胁迫下 的变化趋势明显大于盐胁迫(图 $3 \mathrm{~A}_{1}, 3 \mathrm{~A}_{2}, 3 \mathrm{~B}_{1}, 3 \mathrm{~B}_{2}$ )。 结果表明与盐胁迫相比, 碱胁迫的高 $\mathrm{pH}$ 值进一步干 扰和破坏了根对 $\mathrm{Na}^{+} 、 \mathrm{~K}^{+}$吸收的选择性, 导致体内 $\mathrm{Na}^{+} 、 \mathrm{~K}^{+}$严重失衡, 这是碱胁迫危害大于盐胁迫的一 个主要原因。另外, 碱胁迫下亚麻地上部分中积累 大量 $\mathrm{Na}^{+}$, 致使叶绿体遭到破坏, 叶绿素含量急剧 下降, 导致光合能力降低(图2)。研究还发现, 与根
相比，地上部分中 $\mathrm{K}^{+}$含量保持在较高水平，这说明 亚麻有一个将 $\mathrm{Na}^{+}$控制在根部而将更多的 $\mathrm{K}^{+}$运输到 地上部分的控制适应过程(Munns \& Tester, 2008)。 $\mathrm{Ca}^{2+}$ 对维持细胞膜的稳定性起着重要作用, $\mathrm{Mg}^{2+}$ 是 叶绿素的主要组分, 研究发现某些植物的 $\mathrm{Ca}^{2+}$ 、 $\mathrm{Mg}^{2+}$ 积累受到盐胁迫抑制。本研究结果表明盐、碱 胁迫对亚麻地上部分中 $\mathrm{Ca}^{2+} 、 \mathrm{Mg}^{2+}$ 含量影响不大, 但碱胁迫明显降低其在根中的含量(图 $3 \mathrm{C}_{1}, 3 \mathrm{C}_{2}, 3 \mathrm{D}_{1}$, $\left.3 \mathrm{D}_{2}\right)$ 。 $\mathrm{Ca}^{2+}$ 作为第二信使与盐超敏感性蛋白 $(\mathrm{SOS} 3)$ 结合, 刺激 SOS2 的激酶活性, 排出有毒离子 $\mathrm{Na}^{+}$ ( Zhu, 2003)。但是碱胁迫(高 $\mathrm{pH}$ 值) 导致根周围 $\mathrm{Ca}^{2+}$ 沉淀, $\mathrm{Ca}^{2+}$ 吸收明显受阻, 进而影响 $\mathrm{Na}^{+}$外排, 加速 
了离子毒害作用。另外, $\mathrm{Na}^{+} / \mathrm{H}^{+}$逆向转运蛋白依靠细 胞膜两侧质子梯度排出 $\mathrm{Na}^{+}$, 高 $\mathrm{pH}$ 值导致质子亏缺, 质子梯度难于建立, $\mathrm{Na}^{+}$不能外排, 导致植物体内积 累大量的 $\mathrm{Na}^{+}$(Tester \& Davenport, 2003; 王宁等, 2015)。植物体维持细胞内离子平衡是保障各种代谢 过程正常进行的必要条件, 离子平衡实际上就是阴 阳离子平衡的过程(杨春武等, 2008)。植物吸收阳离 子除了特异性离子通道外, 还有一类非选择性阳离 子通道(Munns \& Tester, 2008), 盐碱胁迫下 $\mathrm{Na}^{+}$大量 进入根部, 可能直接抑制根对其他营养离子的吸收, 导致植物体内离子比率失衡, 如图3中可见盐、碱胁 迫明显降低亚麻 $\mathrm{K}^{+} / \mathrm{Na}^{+} 、 \mathrm{Ca}^{2+} / \mathrm{Na}^{+}$和 $\mathrm{Mg}^{2+} / \mathrm{Na}^{+}$, 并且 碱胁迫下的下降幅度均大于盐胁迫。研究还发现, 盐、碱胁迫造成 $\mathrm{NO}_{3}{ }^{-}$含量降低, 表明大量 $\mathrm{Na}^{+}$积累阻 碍根对 $\mathrm{NO}_{3}{ }^{-}$的吸收, 明显干扰氮吸收或代谢。碱胁 迫强烈干扰无机阴离子积累和分布, 碱胁迫(高 $\mathrm{pH}$ 值)阻碍质子梯度的建立, 从而抑制亚麻根系对阴 离子的吸收, 造成植物营养缺乏, 这可能是碱胁迫 对植物伤害甚于盐胁迫的另一个重要原因。

\section{4 盐、碱胁迫对亚麻有机溶质的影响}

植物在遭受盐胁迫时通常会迅速积累一些有机 溶质作为细胞质中的渗透调节物质, 除渗透调节作 用外, 还可以保护生物大分子并清除自由基(王罗 霞等, 2006)。可溶性糖是植物生命活动最主要的能 源物质, 而且它还具有渗透保护、碳贮存和自由基 清除的功能(Hare et al., 1998)。本研究结果表明盐胁 迫促进可溶性糖的合成和积累, 它不仅参与渗透调 节, 还中和大量的 $\mathrm{Na}^{+}$避免离子伤害, 因此可溶性 糖的积累可以增强亚麻对盐渍环境的耐性(Gao et al., 2001)。但是, 碱胁迫尤其是高碱胁迫抑制了可 溶性糖的积累, 这可能是大量的 $\mathrm{Na}^{+}$和高 $\mathrm{pH}$ 值共同 作用的结果, 碱胁迫破坏了光系统结构, 造成了光 合能力降低, 影响碳同化过程(Guo et al., 2011)。脯 氨酸和甜菜碱作为非毒性的渗透调节物质在植物抗 逆生理中起非常重要的作用(彭志红等, 2002; 张立 新和李生秀, 2004)。盐、碱胁迫都明显刺激了脯氨 酸的积累, 脯氨酸的积累可能是长期离子毒性的代 谢反应, 其含量的增加可能出于维持渗透压和平衡 细胞液泡内无机离子浓度的需要。甜菜碱在盐碱胁 迫下呈现下降的趋势, 这可能是由于 $\mathrm{Na}^{+}$的大量沉 淀, 造成根对 $\mathrm{NO}_{3}$-吸收降低, 阻碍了 $\mathrm{N}$ 代谢的正常 进行, 影响了甜菜碱的合成。从有机酸实验结果可
知, 亚麻可能通过促进有机酸的合成来弥补无机阴 离子的缺失和维护细胞内 $\mathrm{pH}$ 值的稳定(汪建飞和沈 其荣, 2006)。根中合成的有机酸可能是用来调节根 外的 $\mathrm{pH}$ 值, 增加根系对铁等矿质元素的吸收和利用, 进而缓解碱胁迫造成的营养缺失和离子失衡。

基金项目 国家自然科学基金青年基金(31200243)、 国家自然科学基金面上项目(31570328)、国家高技 术研究发展计划 (“863”计划) (2011AA100503)和中 央公益性科研业务费(BSRF 201201)。

\section{参考文献}

Arnon DI (1949). Copper enzymes in isolated chloroplasts phenoloxidases in Beta vulgaris. Plant Physiology, 24, $1-15$.

Ashraf M, Fatima H (2004). Intra-specific variation for salt tolerance in linseed (Linum usitatissimum L). Journal of Agronomy and Crop Science, 173, 193-203.

Bao SD (1981). Analytic Methods for Soil and Agriculture Chemistry. China Agriculture Press, Beijing. 150-160. (in Chinese) [鲍士旦 (1981). 土壤农化分析. 中国农业出 版社, 北京. 150-160.]

Bolhàr-Nordenkampf HR, Long SP, Baker NR, Öquist G, Schreiber U, Lechner EG (1989). Chlorophyll fluorescence as a probe of the photosynthetic competence of leaves in the field: A review of current instrumentation. Functional Ecology, 3, 497-514.

Brugnoli E, Lauteri M (1991). Effects of salinity on stomatal conductance, photosynthetic capacity, and carbon isotope discrimination of salt-tolerant (Gossypium hirsutum L.) and salt-sensitive (Phaseolus vulgaris L.) $\mathrm{C}_{3}$ nonhalophytes. Plant Physiology, 95, 628-635.

Chemikosova SB, Pavlencheva NV, Gur'yanov OP, Gorshkova TA (2006). The effect of soil drought on the phloem fiber development in long-fiber flax. Russian Journal of Plant Physiology, 53, 656-662.

Everard R, Gucci SC, Kann JA, Loescher WH (1994). Gas exchange and carbon partitioning in the leaves of celery (Apium graveolens L.) at various levels of root zone salinity. Plant Physiology, 106, 281-292.

Feng YL, Feng ZL, Cao KF (2001). The protection against photodamage in Amomum villosum Lour. Acta Phytophysiologica Sinica, 27, 483-488. (in Chinese with English abstract) [冯玉龙, 冯志立, 曹坤芳 (2001). 砂仁叶 片光破坏的防御. 植物生理学报, 27, 483-488.]

Gao ZH, Xue YB, Dai JR (2001). cDNA-AFLP analysis reveals that maize resistance to Bipolaris maydis is associated with the induction of multiple defense-related genes. Chinese Science Bulletin, 46, 1454-1458.

Genty B, Briantais JM, Baker NR (1989). The relationship between the quantum yield of photosynthetic electron 
transport and quenching of chlorophyll fluorescence. Biochimica et Biophysica Acta, 990, 87-92.

Gorham J, McDonnell E, WynJones RG (1982). Determination of betaines as ultraviolet-absorbing esters. Analytica Chimica Acta, 138, 277-283.

Guo R, Zhou J, Hao WP, Gong DJ (2011). Germination, growth, photosynthesis and ionic balance in Setaria viridis seedlings subjected to saline and alkaline stress. Canadian Journal of Plant Science, 91, 1077-1088.

Hare PD, Cress WA, Staden JV (1998). Dissecting the roles of osmolyte accumulation during stress. Plant, Cell \& Environment, 21, 535-553.

Jing JH, Ding ZR (1981). Analysis Method of Plant Biochemistry. Science Press, Beijing. 264-267. (in Chinese) [荆家 海, 丁钟荣 (1981). 植物生物化学分析方法. 科学出版 社, 北京. 264-267.]

Kingsbury RW, Epstein E, Peary RW (1984). Physiological responses to salinity in selected lines of wheat. Plant Physiology, 74, 417-423.

Kong LA, Guo HH, Dong XX (2000). A study on ultrastructure of hybrid rumex under salt stress. Acta Prataculturae Sinica, 9(2), 53-57. (in Chinese with English abstract) [孔 令安, 郭洪海, 董晓霞 (2000). 盐胁迫下杂交酸模超微 结构的研究. 草业学报, 9(2), 53-57.]

Li B, Wang ZC, Shun ZG, Chen Y, Yang F (2005). Resources and sustainable resource exploitation of salinized in China. Agricultural Research in the Arid Areas, 23(2), 152-158. (in Chinese with English abstract) [李涁, 王志春, 孙志 高, 陈渊, 杨福 (2005). 中国盐碱地资源与可持续利用 研究. 干旱地区农业研究, 23(2), 152-158.]

Liang HZ, Dou DQ, Feng YL (2004). Diurnal changes in photosynthesis and chlorophyll fluorescence parameters of Amomum villosum Lour. grown under tropical rainforest in rainy, and foggy and cool seasons at Xishuangbanna. Acta Ecologica Sinica, 24, 1421-1429. (in Chinese with English abstract) [梁红柱, 窦德泉, 冯玉龙 (2004). 热带雨林 下砂仁叶片光合作用和叶绿素荧光参数在雾凉季和雨 季的日变化. 生态学报, 24, 1421-1429.]

Lu CM, Qiu NW, Lu QT, Wang BS, Kuang TY (2002). Does saline stress lead to increased susceptibility of photosystem II to photoinhibition and changes in photosynthetic pigment composition in halophyte Suaeda salsa grown outdoors? Plant Science, 163, 1063-1068.

Lu YH, Lam HM, Pi EX, Zhan QL, Tsai S, Wang CM, Kwan YW, Ngai SM (2013). Comparative metabolomics in Glycine max and Glycine soja under salt stress to reveal the phenotypes of their offspring. Journal of Agricultural and Food Chemistry, 61, 8711-8721.

McKenzie RR, Deyholos MK (2011). Effects of plant growth regulator treatments on stem vascular tissue development in linseed (Linum usitatissimum L.). Industrial Crops and
Products, 34, 1119-1127.

Munns R, Tester M (2008). Mechanisms of salinity tolerance. Annual Review of Plant Biology, 59, 651-681.

Peng ZH, Peng KQ, Hu JJ, Xiao LT (2002). Research progress on accumulation of proline under osmotic stress in plants. Chinese Agriculture Science Bulletin, 18(4), 80-83. (in Chinese with English abstract) [彭志红, 彭克勤, 胡家金, 萧浪涛 (2002). 渗透胁迫下植物脯氨酸积累的研究进 展. 中国农学通报, 18(4), 80-83.]

Reddy MP, Vora AB (1986). Changes in pigment composition, Hill reaction activity and saccharides metabolism in bajra (Pennisetum typhoides $\mathrm{S} \& \mathrm{H}$ ) leaves under $\mathrm{NaCl}$ salinity. Photosynthetica, 20, 50-55.

Sa RL, Liu JH, Liu W, Bai JH, Wang ZH (2014). Cationresponsive mechanisms of oats to alkali stress. Acta Agronomica Sinica, 40, 362-368. (in Chinese with English abstract) [萨如拉, 刘景辉, 刘伟, 白健慧, 王占海 (2014). 燕麦对碱胁迫的阳离子响应机制. 作物学报, 40, 362-368.]

Shi DC, Zhao KF (1997). Effects of $\mathrm{NaCl}$ and $\mathrm{Na}_{2} \mathrm{CO}_{3}$ on growth of Puccinellia tenuiflora and on present state of mineral elements in nutrient solution. Acta Prataculturae Sinica, 6, 51-61.

Shi DC, Li YM, Yang GH, Li YD, Zhao KF (2002). A simulation of salt and alkali stress mixed ecological conditions and analysis of their stress factors in the seedlings of Aneurolepidium chinense. Acta Ecological Sinica, 22, 1317-1326. (in Chinese with English abstract) [石德成, 李玉明, 杨国会, 李毅丹, 赵可夫 (2002). 盐碱混合生 态条件的人工模拟及其对羊草胁迫作用因素分析. 生 态学报, 22, 1317-1326.]

Shi DC, Wang D (2005). Effects of various salt-alkaline mixed stresses on Aneurolepidium chinense (Trin.) Kitag. Plant and Soil, 271, 15-26.

Tester M, Davenport RJ (2003). $\mathrm{Na}^{+}$transport and $\mathrm{Na}^{+}$tolerance in higher plants. Annals of Botany, 91, 503-527.

Wang LX, Zhao ZG, Wang SM (2006). Effect of nitric oxide on metabolism of reactive oxygen species and membrane lipid peroxidation in Triticum aestivum leaves under water stress. Aata Prataculturae Sinica, 15(4), 104-108. (in Chinese with English abstract) [王罗霞, 赵志光, 王锁民 (2006). 一氧化氮对水分胁迫下小麦叶片活性氧代谢及 膜脂过氧化的影响. 草业学报, 15(4), 104-108.]

Wang N, Yang J, Huang Q, Su GL, Zhou H, Xu QH, Dong HL, Yan GT (2015). Physiological salinity tolerance mechanism for transport of $\mathrm{K}^{+}$and $\mathrm{Na}^{+}$ions in cotton (Gossypium hirsutum L.) seedlings under salt stress. Cotton Science, 27, 208-215. (in Chinese with English abstract) [王宁，杨杰，黄群，苏桂兰，周红，许庆华，董合林，严 根土 (2015). 盐胁迫下棉花 $\mathrm{K}^{+}$和 $\mathrm{Na}^{+}$离子转运的耐盐性 生理机制. 棉花学报, 27, 208-215.] 
Wang JF, Shen QR (2006). Roles of organic metabolism in plant adaptation to nutrient deficiency and aluminum toxicity stress. Chinese Journal of Applied Ecology, 17, 2210-2216. (in Chinese with English abstract) [汪建飞, 沈其荣 (2006). 有机酸代谢在植物适应养分和铝毒胁 迫中的作用. 应用生态学报, 17, 2210-2216.]

Wu DZ, Shen QF, Cai SG, Chen ZH, Dai F, Zhang GP (2013). Ionomic responses and correlations between elements and metabolites under salt stress in wild and cultivated barley. Plant and Cell Physiology, 54, 1976-1988.

Yan H, Shi DC, Yin SJ, Zhao W (2000). Effects of salinealkaline stress on the contents of nitrogen and several organisms of Aneurolepidium chinense. Journal of Northeast Normal University (Natural Science), 32(3), 47-52. (in Chinese with English abstract) [颜宏, 石德成, 尹尚军, 赵伟 (2000). 盐碱胁迫对羊草体内N及几种有机代谢产 物积累的影响. 东北师范大学学报(自然科学版), 32(3), 47-52.]

Yang C, Shi D, Wang D (2008). Comparative effects of salt stress and alkali stress on growth, osmotic adjustment and ionic balance of an alkali resistant halophyte Suaeda glauca Bge. Plant Growth Regulation, 56, 179-190.

Yang CW, Li CY, Zhang ML, Liu J, Ju M, Shi DC (2008). pH and ion balance in wheat-wheatgrass under salt or alkali stress. Chinese Journal of Applied Ecology, 19, 10001005. (in Chinese with English abstract) [杨春武, 李长有, 张美丽, 刘杰, 鞠沝, 石德成 (2008). 盐、碱胁迫下小冰 麦体内的 $\mathrm{pH}$ 及离子平衡. 应用生态学报, 19, 10001005.]

Zhang LX, Li SX (2004). Research progress on relationships betain and drought/salt resistance of plants. Acta Botanica
Boreali-Occidentalia Sinica, 24, 1765-1771. (in Chinese with English abstract) [张立新, 李生秀 (2004). 甜菜碱 与植物抗旱/盐性研究进展. 西北植物学报, 24 , 1765-1771.]

Zhang JF, Zhang XD, Zhou JX, Liu GH, Li DX (2005). World resources of saline soil and main amelioration measures. Research of Soil and Water Conservation, 12(6), 28-30. (in Chinese with English abstract) [张建锋, 张旭东, 周金 星, 刘国华, 李冬雪 (2005). 世界盐碱地资源及其改良 利用的基本措施. 水土保持研究, 12(6), 28-30.]

Zhang JL, Flowers TJ, Wang SM (2010). Mechanisms of sodium uptake by roots of higher plants. Plant and Soil, 326, 45-60.

Zhang JL, Shi H (2013). Physiological and molecular mechanisms of plant salt tolerance. Photosynthesis Research, $115,1-22$.

Zhang YG, Jianati, Li XS, Zhang HH, Shawulie, Ma HY, Tang $\mathrm{S}$ (2014). Seed germination and physiological responses of Agropyron cristatum 'Tawukumu' under alkali stress. Acta Agrestia Sinica, 22, 783-788. (in Chinese with English abstract) [张一弓, 贾纳提, 李学森, 张荟荟, 沙吾列, 马海燕, 唐森 (2014). 碱胁迫下塔乌库姆冰草的萌发及 生理响应. 草地学报, 22, 783-788.]

Zhu GL, Deng XW, Zuo WN (1983). Determination of free proline in plants. Plant Physiology Communications, 1, 3537. (in Chinese) [朱广廉, 邓兴旺, 左卫能 (1983). 植物 体内游离脯氨酸的测定. 植物生理学通讯, 1, 35-37.]

Zhu JK (2003). Regulation of ion homeostasis under salt stress. Current Opinion in Plant Biology, 6, 441-445.

责任编委: 李凤民 责任编辑: 李 敏 